\title{
The nucleon localization function in static and time-dependent DFT
}

\author{
Bastian Schuetrump ${ }^{1, \star}$ and Chunli Zhang ${ }^{1, \star \star}$ \\ ${ }^{1}$ NSCL/FRIB Laboratory, Michigan State University, East Lansing, Michigan 48824, USA
}

\begin{abstract}
Static and time-dependent density functional theory (DFT) calculations are often used to predict fission fragment distributions or fusion cross sections with great success. However, nuclear shell structure and clusterization effects are usually studied using the density distribution of the nucleons which is a poor indicator of these phenomena. In this work, we employ a measure called the localization function, which was first introduced in chemistry to visualize electronic bonds and recently applied to nuclear physics for light nuclei to reveal $\alpha$-clustering. We show, that the localization function reveals the shell structure of the fragments in fission long before the scission point and also illustrates the shell structure of intermediate states in time-dependent DFT calculations.
\end{abstract}

\section{Introduction}

The emergence of cluster states in atomic nuclei is a universal phenomenon with many implications for both nuclear physics and astrophysics [1-3]. Several factors are contributing to clustering, a complete microscopic understanding has still not been achieved. Cluster configurations are often energetically favorable due to the large binding energy per nucleon in constituent clusters, such as $\alpha$ particles and this argument has often been used to explain properties of $\alpha$-conjugate nuclei [4], cluster emission [5, 6] and fission [7]. Clusters are also coupled to decay channels, which explains $[8,9]$ the occurrence of cluster states at low excitation energies around cluster-decay thresholds [10]. Clustering is of special relevance in the dynamics of the tripple- $\alpha$ reaction [11].

A microscopic description of cluster states requires the use of an advanced many-body framework. A good starting point is density functional theory (DFT) based on a realistic nuclear energy density functional [12]. Within DFT, cluster states have a simple interpretation in terms of quasimolecular states.

The degree of clustering in nuclei is difficult to assess quantitatively in DFT as the single particle wave functions are spread throughout the nuclear volume. Furthermore the single particle states in the Slater determinant are not fixed since the Slater determinant is invariant under unitary transformations. Therefore, in this study, we utilize a measure - dependent on s.p. orbitals, yet invariant under unitary transformation - called localization, originally introduced for the identification of electronic bonds in atomic and molecular systems (see e.g. [13-15]) and has recently been applied to characterize clusters in light nuclei [16].

In this contribution we introduce the localization measure in Sec. 2. In Sec. 3 we show results for heavy nuclei,

\footnotetext{
^e-mail: schutrum@nscl.msu.edu

$\star \star$ e-mail: zhangch@nscl.msu.edu
}

in Sec. 4 we review findings for fissioning nuclei and in Sec. 5 we show first results for time-dependent calculations.

\section{Method}

\subsection{The nucleon localization function}

A localization measure in the DFT framework was first introduced for electronic systems to visualize electronic bonds in [13], referred to as the electronic localization function (ELF). For nuclear systems the localization was first utilized in [16] for light nuclei. For nuclear matter the nucleon localization function (NLF) is based on the inverse conditional probability of finding a nucleon with same spin and isospin in the vicinity of another knowing with certainty that this one is located at $\boldsymbol{r}$. The measure can be expressed through densities and a natural choice for the normalization is the Thomas-Fermi kinetic energy. The resulting dimensionless NLF reads

$$
C_{q \sigma}(\boldsymbol{r})=\left[1+\left(\frac{\tau_{q \sigma} \rho_{q \sigma}-\frac{1}{4}\left|\nabla \rho_{q \sigma}\right|^{2}-\dot{j}_{q \sigma}^{2}}{\rho_{q \sigma} \tau_{q \sigma}^{\mathrm{TF}}}\right)^{2}\right]^{-1}
$$

and contains the densities

$$
\begin{aligned}
\rho_{q \sigma}(\boldsymbol{r}) & =\sum_{\alpha \in q} v_{\alpha}^{2}\left|\psi_{\alpha}(\boldsymbol{r} \sigma)\right|^{2} \\
\tau_{q \sigma}(\boldsymbol{r}) & =\sum_{\alpha \in q} v_{\alpha}^{2}\left|\nabla \psi_{\alpha}(\boldsymbol{r} \sigma)\right|^{2} \\
\boldsymbol{j}_{q \sigma}(\boldsymbol{r}) & =\sum_{\alpha \in q} v_{\alpha}^{2} \operatorname{Im}\left[\psi_{\alpha}^{*}(\boldsymbol{r} \sigma) \nabla \psi_{\alpha}(\boldsymbol{r} \sigma)\right] \\
\boldsymbol{\nabla} \rho_{q \sigma}(\boldsymbol{r}) & =2 \sum_{\alpha \in q} v_{\alpha}^{2} \operatorname{Re}\left[\psi_{\alpha}^{*}(\boldsymbol{r} \sigma) \boldsymbol{\nabla} \psi_{\alpha}(\boldsymbol{r} \sigma)\right] \\
\tau_{q \sigma}^{\mathrm{TF}} & =\frac{3}{5}\left(6 \pi^{2}\right)^{2 / 3} \rho_{q \sigma}^{5 / 3}
\end{aligned}
$$


where $v_{\alpha}^{2}$ is the canonical occupation probability, $q \in\{p, n\}$ labels the isospin and $\sigma \in\{\uparrow, \downarrow\}$ labels the spin or signature symmetry. For the time-independent calculations presented here, time-reversal symmetry is conserved; hence, $\boldsymbol{j}_{q \sigma}$ vanishes. For time-dependent calculations, the spin is in general not a conserved quantity and the signature symmetry has to be taken into account. However, since we are only interested in the total localization of neutrons and protons, we show in this case the localizations $C_{q}$ for average densities $\rho_{q \text { av }}=\left(\rho_{q \uparrow}(\boldsymbol{r})+\rho_{q \downarrow}(\boldsymbol{r})\right) / 2$ and for the other densities respectively.

Two important values for the localization should be mentioned at this point:

$C_{\mathbf{q} \sigma}=\mathbf{0 . 5}$ : Due to the normalization with the ThomasFermi kinetic energy a localization of 0.5 indicates a localization as in a Fermi gas of nuclei.

$C_{\mathbf{q} \sigma}=1.0$ : A localization of one indicates perfect localization, i.e. that the probability of finding another nucleon with same spin and isospin is zero. Perfect localization can be found in an $\alpha$-particle, where only one particle of each spin and isospin combination is present.

In practice especially for light nuclei we found, that if the numerical value the wave function is very small far outside a nucleus, the value of the localization is poorly determined and depends strongly on numerical effects. Since we are only interested in the localization inside a nucleus, we utilize a masking function to suppress the localization outside a nucleus as

$$
C(\boldsymbol{r}) \rightarrow C(\boldsymbol{r}) \rho_{q \sigma}(\boldsymbol{r}) / \max \left[\rho_{q \sigma}(\boldsymbol{r})\right] \quad .
$$

\subsection{DFT engines}

The model of choice for this study is the Skyrme EDF method. In particular we are using the UNEDF1 interaction [17], which was optimized for fission calculations and largely deformed nuclei.

We use two well established EDF solvers for the calculations. For the ground state calculations and constrained fission calculations in Sec. 3 and Sec. 4 we employ the solver HFBTHO [18], based on a deformed harmonic oscillator basis expansion for the wave functions solving the Hartree-Fock-Bogolyubov (HFB) equations including the pairing effect self-consistently.

For the time-dependent calculations in Sec. 5, we employ the solver Sky3D [19] which operates on a cartesian $3 \mathrm{~d}$ grid employing the fast Fourier transform for derivatives. It solves the time-dependent Hartree-Fock equations and includes the pairing effect in the BCS limit with frozen occupations.

\section{Localization in heavy nuclei}

In [16] it has been shown, that the NLF reveals $\alpha$ clustering in light nuclei very clearly. Here, the NLF for heavier nuclei shall be studied.

In Fig. 1 the neutron and proton densities (left panels) as well as the NLF (right panels) of ${ }^{132} \mathrm{Sn}$ are displayed. While the number densities show no structure, except for a small dip in the center of the proton density, the NLF

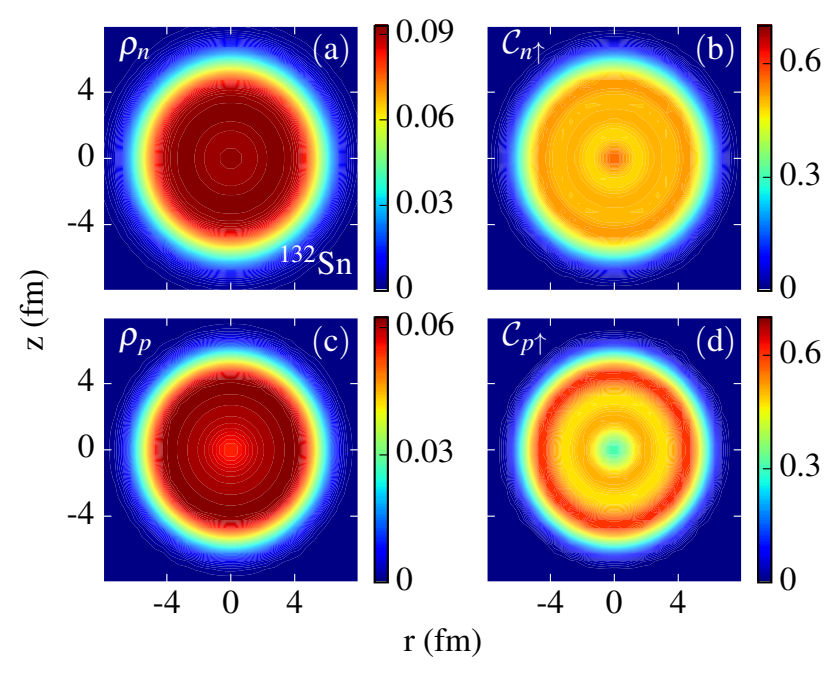

Figure 1: Nucleonic number densities $\left(\mathrm{in}_{\mathrm{fm}}{ }^{-3}\right.$ ) and spatial localizations for the ground state of ${ }^{132} \mathrm{Sn}$. This Figure is taken from [20].

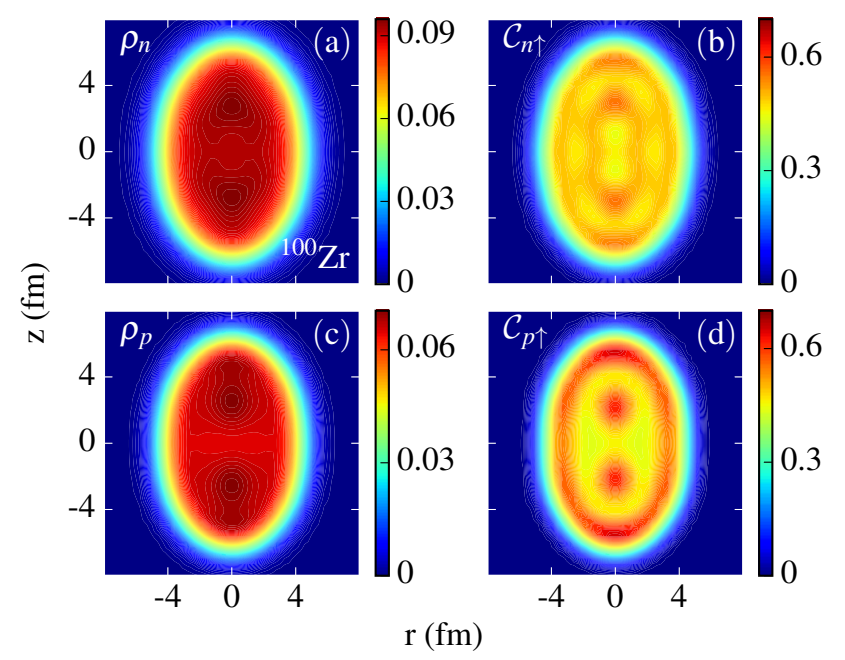

Figure 2: Nucleonic number densities $\left(\mathrm{in}^{-3}\right.$ ) and spatial localizations for the ground state of ${ }^{100} \mathrm{Zr}$. This Figure is taken from [20].

show concentric rings of enhanced localization. The case of ${ }^{132} \mathrm{Sn}$ is particularly interesting, because it is a doubly magic nuclei with one more closed shell for the neutrons. This also manifests in the NLF with an additional enhancement at the center of the neutron NLF. In contrast to electronic systems, the number of closed shells cannot be determined by the number of radial NLF enhancements. The difference is, that in electronic systems the length scale of the shells is proportional to $\sim n^{2}$ where $n$ is the principal quantum number and in nuclear systems it is proportional to $\sim \sqrt{n}$ and also dependent on the angular momentum. Therefore the shells overlap and cannot be clearly distinguished. However, the imprint on the NLF of one more closed shell is clearly visible.

For a second example ${ }^{100} \mathrm{Zr}$ is shown in Fig. 2. This nucleus does not have a magic number of either nucleons and is prolately deformed. The proton NLF reveals two 


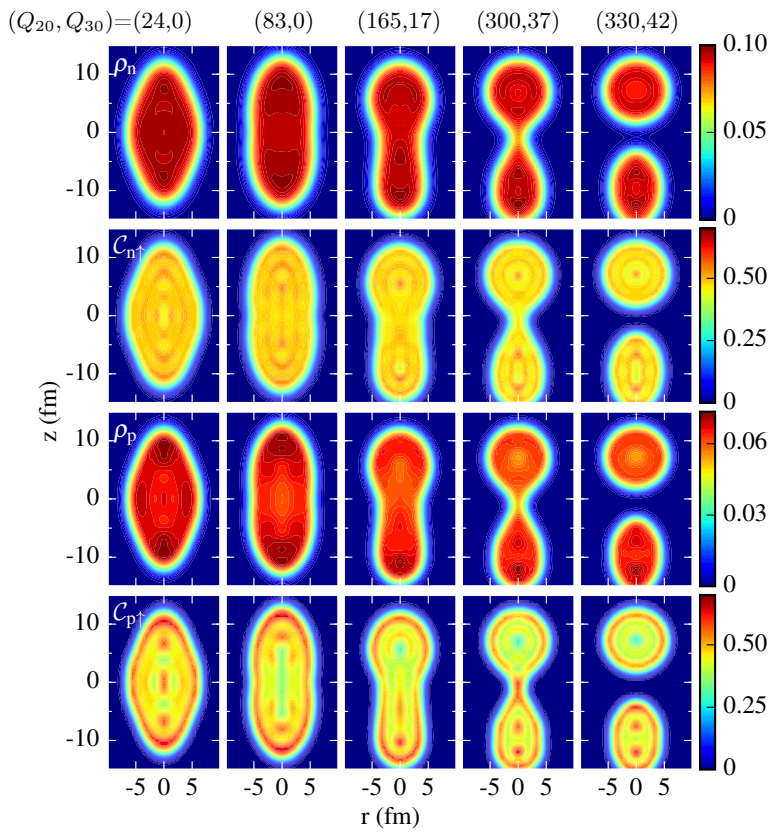

Figure 3: Nucleonic number densities (in $\mathrm{fm}^{-3}$ ) and spatial localizations for ${ }^{232} \mathrm{Th}$ for five configurations along the fission pathway. This Figure is taken from [20]. The deformations are marked at the top of each column with in units of $b$ and $b^{3 / 2}$, respectively.

centers and one ring of enhanced localization, whereas the neutron NLF exhibits a two ring structure. Also here the 20 additional neutrons leave a strong imprint on the localization.

\section{Localization for fission calculations}

As a next step we study the localization during fission in heavy nuclei. As an example we take the asymmetric fission of ${ }^{232} \mathrm{Th}$. We consider the fission pathway which has been calculated in [21] and plot the densities and NLF for five points on this pathway in Fig 3. This pathway leads to the two fragments ${ }^{132} \mathrm{Sn}$ and ${ }^{100} \mathrm{Zr}$ which were discussed in Sec. 3. This will make it possible to compare the NLFs of the compound nucleus to those of the fragments. For that purpose we performed static calculations with constraints on the quadrupole moment $Q_{20}$ which quantifies the elongation and the octupole moment $Q_{30}$ which quantifies the mass asymmetry as marked at the top of the figure. Rotational symmetry along the $\mathrm{z}$-axis is assumed. The state which is displayed in the left column corresponds to the ground state. The next state is the metastable fission isomer. The third state is a state across the barrier which has already a non-zero mass asymmetry deformed. The fourth state is around the scission point and the fifth state shows already separated fragments.

While the first two compact configurations have a very distinct structure in the NLF, they do not resemble the fragments. In contrast, the first configuration on the asymmetric part of the path shows already the structure of the fragments. It is also worth to notice, that in the neck region,

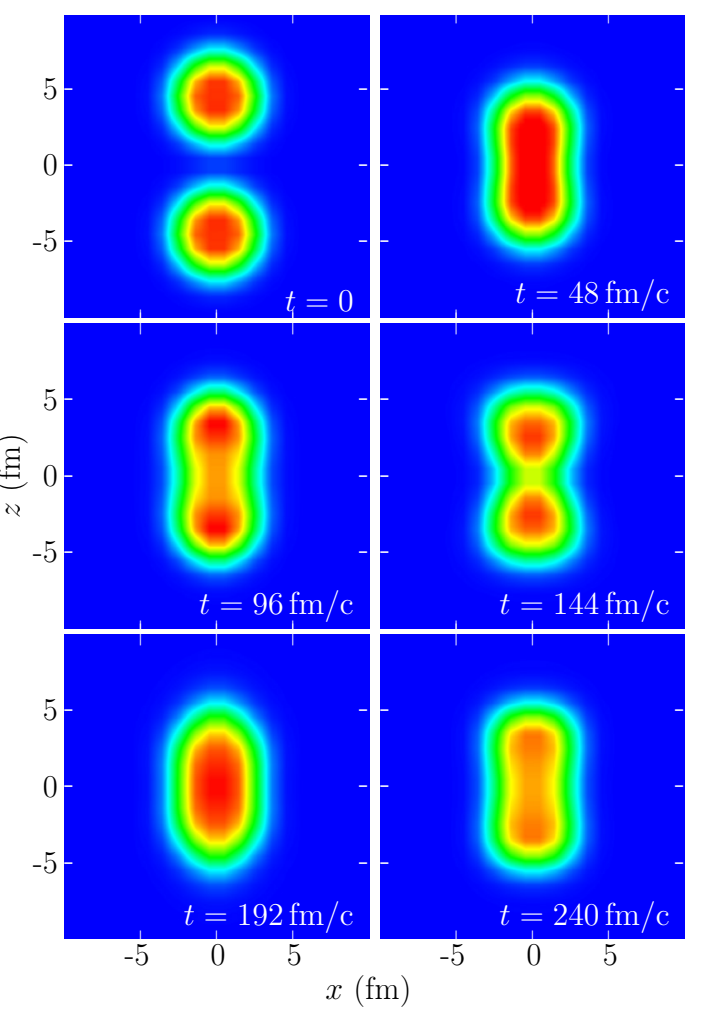

Figure 4: Snapshots of the time evolution of the nucleonic number densities (in $\mathrm{fm}^{-3}$ ) of the central collision of two ${ }^{16} \mathrm{O}$ nuclei with $E_{\mathrm{cm}}=40 \mathrm{MeV}$.

where both fragments overlap, the NLF has values around 0.5 . This indicates, that in this region there is not a distinct shell structure dominant and the structure is similar to a Fermi gas. The density however does not show any resemblance to a specific fragment and the neck contains still a large number of nucleons which makes it impossible to predict the fragments.

The fourth configuration shows even more clearly the NLF of the fragments. The neck is already very small and especially for the neutrons an enhanced localization is visible. This enhancement is the result of very few particles which are present in the neck so the possibility of finding a second with same spin and isospin is very small. The fifth configuration finally exhibits the exact structure of the fragments.

\section{Localization in time-dependent DFT}

In this section we focus on first time-dependent results for the NLF. As a test case we picked the symmetric collision between two ${ }^{16} \mathrm{O}$ nuclei. First we consider a central collision with a center-of-mass energy of $40 \mathrm{MeV}$.

The density distribution is shown in Fig. 4. In practice the calculation is performed in a $32 \mathrm{fm} \times 32 \mathrm{fm} \times 32 \mathrm{fm}$ box with a larger initial separation as shown in the Figure. We show here only a sector in space and time. In this collision the two oxygen form a compound nucleus. At $t=144 \mathrm{fm} / \mathrm{c}$ the compound has the largest deformation 


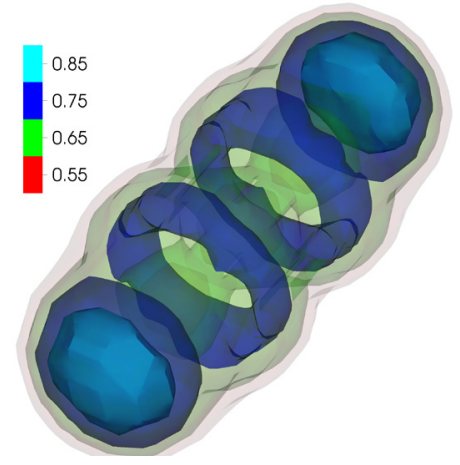

(a) $t=192 \mathrm{fm} / \mathrm{c}$

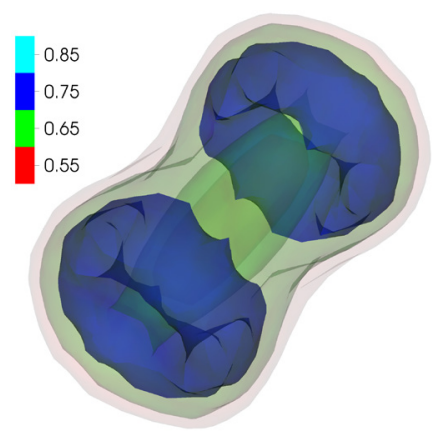

(b) $t=240 \mathrm{fm} / \mathrm{c}$

Figure 5: Snapshots of the time-dependent evolution of the neutron NLF of the central collision of two ${ }^{16} \mathrm{O}$ nuclei with $E_{\mathrm{cm}}=40 \mathrm{MeV}$.

and the two oxygen are again visible in the density. Afterwards the compound oscillates between two configurations which are shown in the lower panels at $t=192 \mathrm{fm} / \mathrm{c}$ and $t=240 \mathrm{fm} / \mathrm{c}$.

In the evolution of the NLF much more structure is visible. In Fig 5 two configurations of the neutron NLF are shown, corresponding to the configurations in the lower panel of Fig. 4. Here, we only show the neutron NLF, since for light isospin symmetric nuclei the difference between the neutron and proton NLF stemming from the small Coulomb contribution is neglectable for our purpose.

In the configuration at $t=192 \mathrm{fm} / \mathrm{c}$ large NLF values at the tips of the compound are visible. These are strong indicators of $\alpha$-clusters. The rings of enhanced localization in between are often indicators for a ${ }^{12} C$-like shell structure. It can be regarded as an ${ }^{16} \mathrm{O}$ nucleus without one of the p-shells which create a hole in the spherical NLF in one direction. The particle content of the regions where the rings are present confirms this fact. The NLF ring-like configuration is not the ground state of a ${ }^{12} \mathrm{C}$ nucleus but an excited state. We interpret this state of the ${ }^{32} \mathrm{~S}$ compound nucleus as a $\alpha-{ }^{12} \mathrm{C}-{ }^{12} \mathrm{C}-\alpha$. Such a ring can also be found in the ground states of nuclei as e.g. in ${ }^{20} \mathrm{Ne}$. In [16] ${ }^{20} \mathrm{Ne}$ was interpreted as a quasi-molecular $\alpha-{ }^{12} \mathrm{C}-\alpha$ configuration.
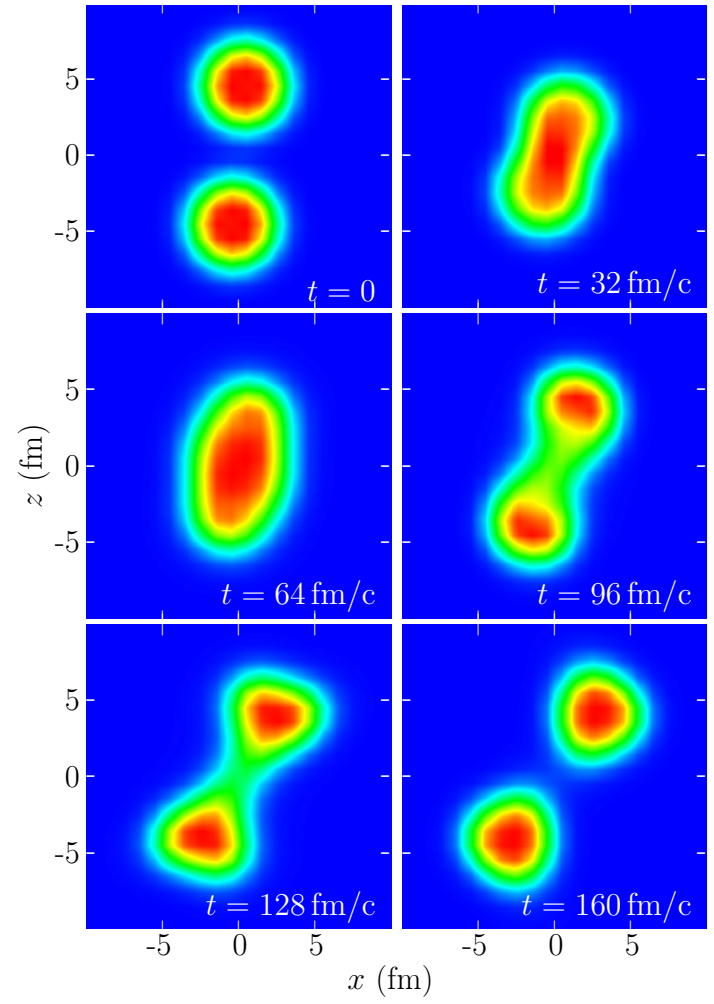

Figure 6: Same as Fig. 4 but for $E_{\mathrm{cm}}=100 \mathrm{MeV}$ and $b=2 \mathrm{fm}$

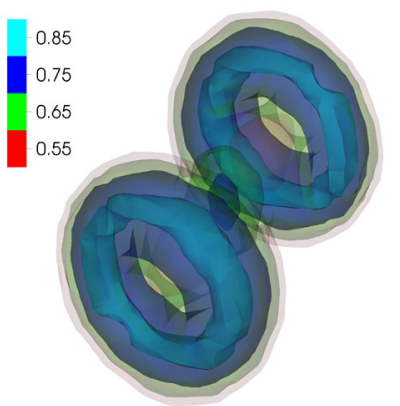

Figure 7: Same as Fig. 5 but for $E_{\mathrm{cm}}=100 \mathrm{MeV}$ and $b=2 \mathrm{fm}$ at time $t=96 \mathrm{fm} / \mathrm{c}$.

In the second configuration at $t=240 \mathrm{fm} / \mathrm{c}$ the enhancements at the tips have vanished. The separation of the two rings however increased. This suggests, that the structure has changed to two ${ }^{12} \mathrm{C}$-like clusters at the tips. The remaining nuclei have been shifted into the interior. They do not appear as $\alpha$-clusters, but the nucleons are delocalized between the rings and therefore do not show an enhancement in the localization.

Figure 6 displays the time evolution of the density distribution of a noncentral collision with an impact parameter of $b=2 \mathrm{fm}$ and a center-of-mass energy of $E_{\mathrm{cm}}=$ $100 \mathrm{MeV}$. After the two fragments form a compound nucleus the two fragments rotate connected with a neck and then separate around $t=160 \mathrm{fm} / \mathrm{c}$. 
The most interesting configuration for the NLF is here the configuration at $t=96 \mathrm{fm} / \mathrm{c}$ shown in Fig. 7. The two ${ }^{12} \mathrm{C}$ rings are present, similar to the central case, but an enhancement inside the neck is also present. This enhancement results again from the small possibility of finding a second particle with same spin and isospin inside the small neck region. Afterwards, the spherical enhancement of the ${ }^{16} \mathrm{O}$ fragments appear again when the neck vanishes.

\section{Conclusion}

In our work, we expand the use of the localization from light nuclei, where mainly $\alpha$-clusters can be exhibited, to heavy nuclei, where imprints of the shell structure can be observed. We show at the example of ${ }^{132} \mathrm{Sn}$, that the imprint of closed shells are clearly visible in the NLF, although the length scale of the shells in a nucleus is proportional to $\sim \sqrt{n}$ and also dependent on the angular momentum and thus can overlap. The example of ${ }^{100} \mathrm{Zr}$ demonstrates the impact of open shells.

Furthermore we expose the usefulness of the NLF for fission calculations. The NLF in the asymmetric decay of ${ }^{232} \mathrm{Th}$ illustrates, that the structure of the fragments is visible in the calculations long before the scission point. We suggest, that this method can therefore be used in such fission calculations for an earlier identification.

The NLF in time-dependent calculations reveals, that a strong imprint of the shell structure is still visible in highly excited systems. The study of the central collision of two ${ }^{16} \mathrm{O}$ nuclei demonstrates, that the states of the ${ }^{32} \mathrm{~S}$ compound nucleus which are occupied are of the nature of $\alpha$ ${ }^{12} \mathrm{C}-{ }^{12} \mathrm{C}-\alpha$ and another state with ${ }^{12} \mathrm{C}$ at the tips and the remaining nucleons in the center. It shows clearly, that quantum calculations as performed in this work exhibit much more information than a liquid drop picture and that the shell structure is immensely important. The non-central fusion fission reaction suggests, that intermediate states in reactions can be very important and the NLF provides a powerful access.

With the case of the collision between two ${ }^{16} \mathrm{O}$ nuclei the study of the NLF is at the very beginning. We intend to expand the study to a variety of medium mass nuclei. The effect of isospin asymmetry has still to be investigated. We also plan to perform time-dependent calculations with pairing in the BCS limit.

Useful discussions with P.-G. Reinhard, N. Schunck, and A.S. Umar are gratefully acknowledged. This work was supported by the U.S. Department of Energy, Office of Science under Award Numbers DOE-DE-NA0002847 (the Stewardship Science Academic Alliances program), DE-SC0013365 (Michigan State University), and DE-SC0008511 (NUCLEI SciDAC-3 collaboration). An award of computer time was provided by the Institute for Cyber-Enabled Research at Michigan State University. We also used computational resource provided by the National Energy Research Scientific Computing Center (NERSC).

\section{References}

[1] C. Beck, ed., Clusters in Nuclei, vol 1, Vol. 818 of Lecture Notes in Physics (Springer, Berlin, Heidelberg, 2010)

[2] C. Beck, ed., Clusters in Nuclei, vol 2, Vol. 848 of Lecture Notes in Physics (Springer, Berlin, Heidelberg, 2012)

[3] C. Beck, ed., Clusters in Nuclei, vol 3, Vol. 875 of Lecture Notes in Physics (Springer, Berlin, Heidelberg, 2014)

[4] L.R. Hafstad, E. Teller, Phys. Rev. 54, 681 (1938)

[5] H.J. Rose, G.A. Jones, Nature 307, 245 (1984)

[6] D. Aleksandrov, A. Belyatskii, Y. Glukhov, E. Nikol'Skií, B. Novatskiì, A. Ogloblin, D. Stepanov, ZhETF Pisma Redaktsiiu 40, 152 (1984)

[7] S. Bjørnholm, J.E. Lynn, Rev. Mod. Phys. 52, 725 (1980)

[8] J. Okołowicz, M. Płoszajczak, W. Nazarewicz, Prog. Theor. Phys. Suppl. 196, 230 (2012)

[9] J. Okołowicz, W. Nazarewicz, M. Płoszajczak, Fortschr. Phys. 61, 66 (2013)

[10] K. Ikeda, N. Takigawa, H. Horiuchi, Prog. Theor. Phys. Suppl. E68, 464 (1968)

[11] A.S. Umar, J.A. Maruhn, N. Itagaki, V.E. Oberacker, Phys. Rev. Lett. 104, 212503 (2010)

[12] M. Bender, P.-H. Heenen, P.-G. Reinhard, Rev. Mod. Phys. 75, 121 (2003)

[13] A.D. Becke, K.E. Edgecombe, J Chem. Phys. 92, 5397 (1990)

[14] M. Kohout, Int. J. Quantum Chem. 97, 651 (2004)

[15] J. Poater, D. M., M. Solà, B. Silvi, Chem. Rev. 105, 3911 (2005)

[16] P.-G. Reinhard, J.A. Maruhn, A.S. Umar, V.E. Oberacker, Phys. Rev. C 83, 34312 (2011)

[17] M. Kortelainen, J. McDonnell, W. Nazarewicz, P.-G. Reinhard, J. Sarich, N. Schunck, M.V. Stoitsov, S.M. Wild, Physical Review C 85, 024304 (2012)

[18] M.V. Stoitsov, N. Schunck, M. Kortelainen, N. Michel, H. Nam, E. Olsen, J. Sarich, S. Wild, Computer Physics Communications 184, 1592 (2013)

[19] J.A. Maruhn, P.-G. Reinhard, P.D. Stevenson, A.S. Umar, Comput. Phys. Commun. 185, 2195 (2014)

[20] C.L. Zhang, B. Schuetrumpf, W. Nazarewicz, Physical Review C 94, 064323 (2016)

[21] J.D. McDonnell, W. Nazarewicz, J.A. Sheikh, Physical Review C 87, 054327 (2013) 